\title{
Probing DNA - Transcription Factor Interactions Using Single-Molecule Fluorescence Detection in Nanofluidic Devices
}

\author{
Mattia Fontana, Šarune Ivanovaitè, Simon Lindhoud, Elmar van der Wijk, \\ Klaus Mathwig, Willy van den Berg, Dolf Weijers, and Johannes Hohlbein*
}

Single-molecule fluorescence detection offers powerful ways to study biomolecules and their complex interactions. Here, nanofluidic devices and camerabased, single-molecule Förster resonance energy transfer (smFRET) detection are combined to study the interactions between plant transcription factors of the auxin response factor (ARF) family and DNA oligonucleotides that contain target DNA response elements. In particular, it is shown that the binding of the unlabeled ARF DNA binding domain (ARF-DBD) to donor and acceptor labeled DNA oligonucleotides can be detected by changes in the FRET efficiency and changes in the diffusion coefficient of the DNA. In addition, this data on fluorescently labeled ARF-DBDs suggest that, at nanomolar concentrations, ARF-DBDs are exclusively present as monomers. In general, the fluidic framework of freely diffusing molecules minimizes potential surfaceinduced artifacts, enables high-throughput measurements, and proved to be instrumental in shedding more light on the interactions between ARF-DBDs monomers and between ARF-DBDs and their DNA response element.

conformational changes of biomolecules with nanometer precision. ${ }^{[3-5]}$

Traditionally, single-molecule fluorescence detection (SMFD) can be carried out either on a confocal microscope, which uses one or more avalanche photodiodes as point detectors, or on a wide-field microscope used in total-internal reflection fluorescence (TIRF) mode, which uses emCCD or sCMOS cameras to monitor hundreds of molecules in parallel.[6] SMFD of freediffusing molecules on a confocal microscope allows for high time resolution (typically $\mu \mathrm{s}$ ) at the expense of throughput and short observation times while SMFD of surface-immobilized molecules on a TIRF microscope displays a somehow complementary behavior with lower time resolution (typically $\mathrm{ms}^{\left[{ }^{[]]}\right.}$) compensated by high throughput and long observation times.

\section{Introduction}

Single-molecule techniques are playing an increasingly important role in the investigation of properties and dynamics of proteins and biomolecular complexes, thanks to their ability to resolve inter- and intramolecular heterogeneity. ${ }^{[1]}$ In particular, fluorescence-based techniques became widely employed thanks to their ease of use ${ }^{[2]}$ and, in the case of single-molecule Förster resonance energy transfer (smFRET), their ability to resolve
During the past decade, different frameworks were proposed to overcome the limitations imposed by these traditional implementations of SMFD. For confocal microscopy, the main focus has been prolonging the observation times ${ }^{[8-13]}$ while in TIRF-based applications, the aim was to eliminate the need of sample immobilization. ${ }^{[1-16]}$ Performing SMFD experiments on a TIRF microscope without immobilization allows to minimize surface-induced artifacts whilst maintaining the high throughput inherent to camera-based detection schemes.
M. Fontana, Š. Ivanovaité, E. van der Wijk

Laboratory of Biophysics

Wageningen University and Research

Stippeneng 4, Wageningen 6708 WE, The Netherlands

M. Fontana, S. Lindhoud, E. van der Wijk, W. van den Berg, D. Weijers

Laboratory of Biochemistry

Wageningen University and Research

Stippeneng 4, Wageningen 6708 WE, The Netherlands

The ORCID identification number(s) for the author(s) of this article can be found under https://doi.org/10.1002/adbi.202100953.

(C) 2021 The Authors. Advanced Biology published by Wiley-VCH GmbH. This is an open access article under the terms of the Creative Commons Attribution License, which permits use, distribution and reproduction in any medium, provided the original work is properly cited.

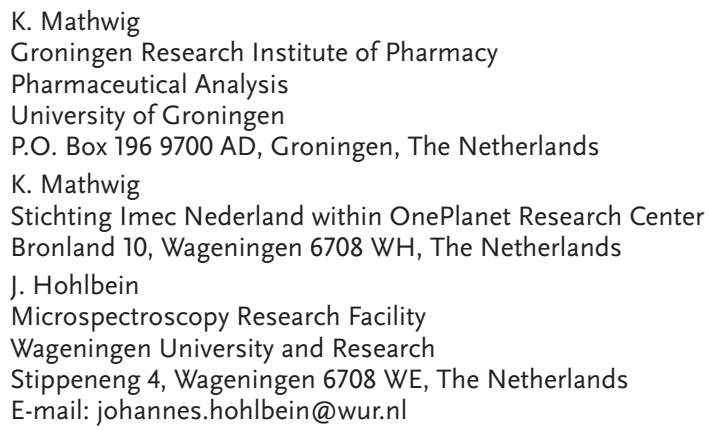

DOI: 10.1002/adbi.202100953 
Here, we utilize a glass-made fluidic device ${ }^{[15]}$ on a TIRF microscope to study members of the transcription factor family of auxin response factors (ARFs) and their interaction with their DNA response element. The ARF family of transcription factors is involved in the response to auxin, a plant hormone that regulates many developmental processes. ${ }^{[17-19]}$ While much is known about their binding preferences, a detailed understanding of the binding process is still lacking. We were able to detect the interaction between ARF DNA binding domain (ARF-DBD) with a doubly labeled DNA construct containing the response element by monitoring the change in its FRET signature and the diffusion coefficient upon DNA binding. We then studied fluorescently labeled ARF-DBDs to show that they are present in their monomeric form at the nanomolar concentrations used in our experiments, which contrast with the dimeric form portrayed in multiple crystal structures. ${ }^{[18,20]}$

\section{Experimental Section}

\subsection{Device Fabrication, Surface Passivation, and Cleaning}

The fluidic devices were fabricated by Micronit (Micronit Microtechnologies B.V., The Netherlands) following the same methodology and design described previously. ${ }^{[15]}$ To prevent non-specific adsorption of the analytes, passivating cleaned nanochannels using polyethylene glycol (mPEG, $M_{W}=5000 \mathrm{Da}$, Laysan Bio Inc., USA) were opted as follows. First, the channels were washed with acetone and then incubated for $5 \mathrm{~min}$ with a 50:1 acetone:Vectabond solution; then, channels were washed with acetone $(1 \times)$ followed up by MilliQ $(3 \times)$ and incubated with a solution of MPEG in MOPS buffer(pH 7.5) for $3 \mathrm{~h}$. The pegylated channels were then washed with PBS buffer and stored in a wet chamber at $4^{\circ}$. After the experiments, the devices were washed by flowing and incubating a solution of 50:1 MilliQ:Hellmanex III (Hellma, Germany). After rinsing with MilliQ, the devices were burned in a furnace at $500^{\circ}$.

\subsection{Protein Expression and Purification}

The DBDs of MpARF1, MpARF2, AtARF1, and AtARF5 were amplified and cloned in a modified pTWIN1 vector. The expression and purification of recombinant ARF-DBDs were performed as previously described. ${ }^{[18,20]}$

\subsection{Protein Labeling}

The stochastic labeling of surface-exposed lysine residues was carried out using a labeling buffer composed of 20 parts of solution 1 (274 mM NaCl, $5.4 \mathrm{mM} \mathrm{KCl}$, and $10 \mathrm{mM}$ phosphate) and one part of solution $2\left(0.2 \mathrm{M} \mathrm{NaHCO}^{3}, \mathrm{pH}\right.$ adjusted to 9 using $\mathrm{NaOH})$; the $\mathrm{pH}$ of the final labeling buffer was adjusted to 8.3 using solutions 1 or 2 . Buffer exchange was carried out by two consecutive runs on Zeba Spin Desalting Columns 7MWCO (Thermo Fisher Scientific, USA) that was equilibrated in labeling buffer. For labeling ARF5-DBD, $60 \mu \mathrm{L}$ of a $3.5 \mathrm{mg} \mathrm{mL}^{-1}$ ARF5-DBD solution was supplemented with $12 \mu \mathrm{L}$ of $800 \mu \mathrm{M}$
DNA duplex and $1 \mathrm{mM}$ TCEP (final protein concentration: $65 \mu \mathrm{M}$, final DNA concentration: $133 \mu \mathrm{M}) .5 \mu \mathrm{L}$ of $10 \mathrm{mM}$ label was added. The labeling reaction was carried out overnight at $4^{\circ}$; then, the labeled protein was separated from free dye on a PD-10 desalting column (Cytiva, USA) that was equilibrated in PBS supplemented with $5 \mathrm{mM}$ DTT. For ARF1-DBD, the labeling was performed as described for ARF5-DBD but in absence of the DNA duplex.

\subsection{DNA Constructs and Imaging Conditions}

The design of the doubly-labeled DNA construct was similar to the one previously used by the authors to determine the binding affinity of the DNA binding domains (DBDs) of Marchantia polymorpha ARF 1 and 2. ${ }^{[20]}$ The construct contained two high affinity binding sites for ARF (TGTCGG) in an inverted topology with a spacer of 7 bp (IR7) and was labeled with a FRET pair featuring Cy3B as the donor and ATTO647N as the acceptor fluorophore. Samples were measured in PBS buffer (137 mM NaCl, $2.7 \mathrm{mM} \mathrm{KCl}$, and $10 \mathrm{mM}$ phosphate). In the experiment presented in Figures $2 \mathrm{c}$ and $3 \mathrm{c}$, an oxygen scavenger system was added to the solution ${ }^{[21,22]}$ for a final concentration of $1 \mathrm{mM}$ Trolox, $1 \%$ gloxy, and 1\% glucose.

\subsection{Accessible Volume Simulations}

The web server 3D-DART ${ }^{[23]}$ was used to first model a straight, standard B-DNA structure for the oligo used in the smFRET experiments. A second, bent oligo was modeled using the geometrical information extracted from the short DNA present in the crystal structure of MpARF2 (PDB ID: 6SDG). The bent oligo was then rigid-docked in place of the short oligo that was present in the crystal structure. Both oligos were then used as starting points to model the accessible volumes of the FRET pair, as well as the average distances between the fluorophores, using the FRET-restrained positioning and screening software. ${ }^{[24]}$ The accessible volume was defined by the region of space that each dye could explore given its own geometry, the geometry of the linker, and the attachment point on the biomolecule (see Table 1).

\subsection{Single-Molecule Detection and Tracking}

Single-molecule detection was performed using a TIRF microscope (previously described ${ }^{[15]}$ ) equipped with a fiber-coupled laser engine (Omicron, Germany). The triggering of the lasers and the camera as well as the laser intensities were controlled

Table 1. AV simulations: Geometrical parameters of the dyes taken from ref. [25].

\begin{tabular}{lcccccc}
\hline Dye & Linker & $\begin{array}{c}L \text { (length) } \\
{[\AA \AA]}\end{array}$ & $\begin{array}{c}L \text { (width) } \\
{[\AA \AA]}\end{array}$ & $\begin{array}{c}R_{1} \\
{[\AA \AA]}\end{array}$ & $\begin{array}{c}R_{2} \\
{[\AA \AA]}\end{array}$ & $\begin{array}{c}R_{3} \\
{[\AA \AA]}\end{array}$ \\
\hline \multirow{2}{*}{ Cy3B } & C6-NHS & 14.2 & 4.5 & 8.2 & 3.3 & 2.2 \\
ATTO647N & C6-NHS & 17.8 & 4.5 & 7.4 & 4.8 & 2.6 \\
\hline
\end{tabular}


by a home-written LabVIEW program. The power of the lasers was set to $100 \%$ which results in a measured laser power at the fiber output of $280 \mathrm{~mW}$ for the green excitation (561 nm) and $140 \mathrm{~mW}$ for the red excitation $(638 \mathrm{~nm})$. The illumination was based on the stroboscopic alternating-laser excitation $\left(\right.$ sALEX $\left.^{[7]}\right)$ scheme with laser pulses of $1.5 \mathrm{~ms}$ in a frame time of $10 \mathrm{~ms}$. Green and red pulses of two subsequent frames were placed back-to-back to facilitate tracking between these coupled frames. Particles were localized using a modified version of GaussStorm, ${ }^{[26]}$ (available at https://kapanidis.web.ox.ac.uk/ software) and tracked using Maria Kilfoil's MATLAB porting of a tracking algorithm developed in IDL. ${ }^{27]}$ For samples containing only one fluorophore (e.g., singly-labeled proteins), a scheme based on stroboscopic back-to-back illumination was applied to the single excitation laser. Per condition, we acquired four movies of 4000 frames for a total acquisition time of $160 \mathrm{~s}$.

\subsection{FRET and ALEX}

The apparent FRET efficiency $E^{*}$ was calculated from the emission intensities of donor and acceptor after donor excitation (denoted as DD and DA) according to

$E^{*}=\frac{\mathrm{DA}}{\mathrm{DD}+\mathrm{DA}}$

Moreover, alternating laser excitation (ALEX) scheme was used; in this scheme every frame where the donor is excited was followed by one of direct excitation of the acceptor fluorophore using a second laser resulting in a third photon stream (AA) for each molecule. ${ }^{[4,28,29]}$ This additional information allows for calculating the apparent stoichiometry ratio $S^{*}$, defined as

$S^{*}=\frac{\mathrm{DD}+\mathrm{DA}}{\mathrm{DD}+\mathrm{DA}+\mathrm{AA}}$

$S^{*}$ can be used to filter molecules: molecules with a stoichiometry close to 0 have no photoactive donor whereas molecules with a stoichiometry close to 1 have no photoactive acceptor. A stoichiometry around 0.5 represented molecules having both photoactive donor and acceptor. The fit of the FRET efficiency distribution was performed using two Gaussian, each defined by three parameters: amplitude, mean, and variance. Amplitudes and variances were left unconstrained. The means were constrained around the values of the respective majority population (e.g., high FRET in Figure 2c top and low FRET in Figure 2c bottom).

\subsection{Particle Displacements Analysis}

The particle displacements between two back-to-back illuminated frames were obtained using 2D Gaussian fitting of the imaged point spread functions as described previously, ${ }^{[15]}$ (see also Figures S1 and S2, Supporting Information). From the variance of the 1D Gaussian distributions of all 1D displacements in either $x$-, or $\gamma$-direction ( $\sigma^{2}$, also called mean-square-displacement), the mean particle diffusion coefficient $D$ was calculated using
$D=\frac{\sigma^{2}}{2 t}$

here, $t$ is the time between the mean time of two back-to-back illuminations $(1.5 \mathrm{~ms})$. In case the displacements were calculated along the direction of the flow, the mean flow speed $(\langle v\rangle)$ was determined by the mean $(\mu)$ of their distribution

$\langle v\rangle=\frac{\mu}{t}$

We note that Equations (3) and (4) do not take into consideration the uncertainty associated with the localization. While Equation (4) holds true, calculating the diffusion coefficient from Equation (3) requires knowledge on the variance that was generated by the localization uncertainty (of both localizations) and its subtraction from the total variance to isolate the component that was generated by the Brownian motion. Consequently, diffusion coefficients calculated without accounting for the localization uncertainty will overestimate the real value.

\subsection{Calculation of Theoretical Diffusion Coefficients}

The predicted diffusion coefficients presented in this chapter were calculated using HYDROPRO. ${ }^{[30]}$ This software uses the crystal structure of a macromolecule to estimate its diffusion coefficient. The calculations were performed loading the pdb file of the protein or complex of interest, setting the temperature to $20^{\circ}$ and the viscosity of the solution to $1 \mathrm{cP}$; the calculations were performed in Mode 1 (shell-mode from atomic-level) using a radius of the atomic elements of $2.84 \AA$.

\subsection{Statistical Analysis}

The displacement distributions in Figures 3 and 4 represent all the displacements retrieved from the four movies of each dataset; the error values reported in the main text represent the standard error of the mean calculated from the individual movies of each dataset (see also Supporting Information).

\section{Results}

\subsection{SMFD in Parallel Nanochannels}

Detecting non-immobilized, nanometer-sized molecules in fluidic devices using camera-based microscopy is challenging as the movement of the molecule due to diffusion and advection within the time of a single camera frame results in the spreading of emitted photons over many pixels. Given the already limited photon budget of single emitters, the movement further decreases the signal-to-noise ratio.

To mitigate the motion blur caused by advection, the fluidic design should provide sufficiently slow flow speeds while still allowing for fast exchange of sample solutions. A convenient way of implementing such characteristics is to use parallel flow 

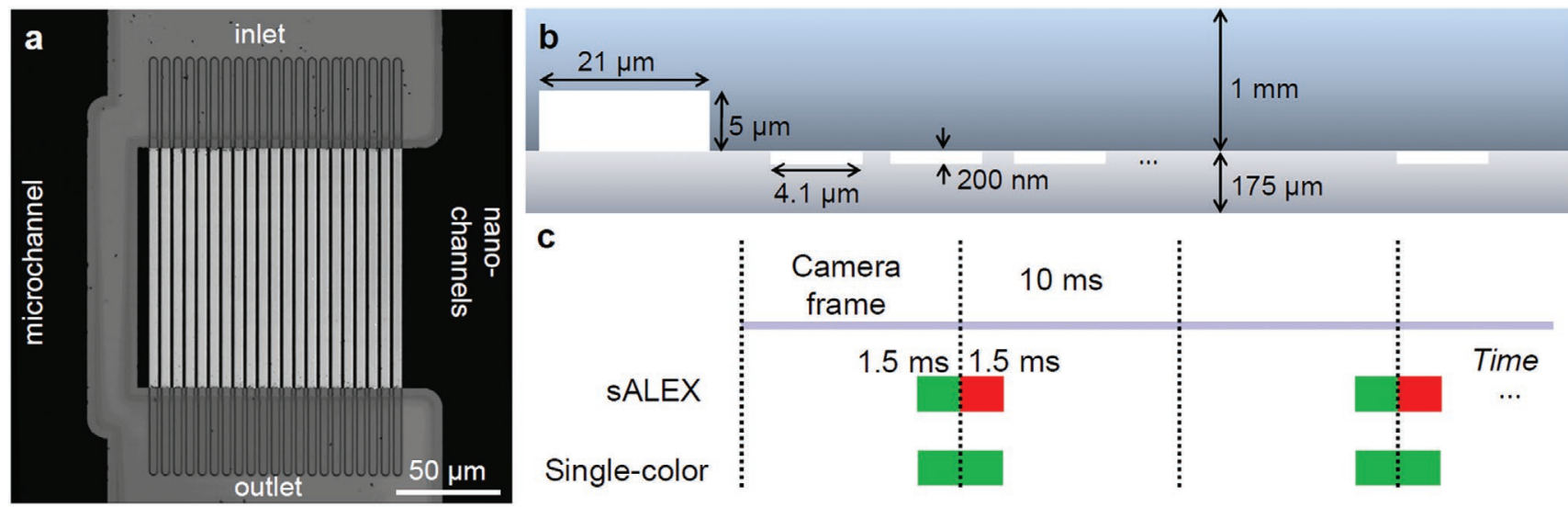

Figure 1. Design of the fluidic device and excitation (Adapted with permission. ${ }^{[15]}$ Copyright 2019, Royal Society of Chemistry). a) High-resolution confocal reflection scan of the parallel nanochannels device; both the array of imaging nanochannels $(I \times w \times h: 120$ by 4.1 by $0.2 \mu \mathrm{m})$ and the bypassing microchannel $(I \times w \times h: 120$ by 21 by $5 \mu \mathrm{m})$ are visible. b) Schematic cross-section of the parallel channel array, showing the dimensions of the microchannel and the nanochannels. The microchannel has a larger cross-section thereby causing lower resistance to the fluid. As a result, most of the sample liquid passes through the microchannel minimizing both the speed of the analyte inside the nanochannels and the time it takes for the analyte to reach the nanochannels. c) Schematic representation of the laser excitation schemes. The concept of stroboscopic illumination is used to reduce the motion blur of single-particle localization while the excitation of two successive frames is placed back-to-back to facilitate tracking and allowing for higher concentrations of fluorescent molecules to be analyzed.

control (PFC). ${ }^{[31,32]}$ In our implementation of PFC, ${ }^{[15]}$ the flow coming from a syringe pump $\left(\approx 10 \mu \mathrm{L} \mathrm{h}^{-1}\right)$ is divided between the imaging channels (i.e., nanochannels) and a bypassing microchannel (Figure 1a,b) such that most of the liquid passes through the microchannel due to its lower hydraulic resistance thereby reducing the fluid velocity inside the nanochannels by several orders of magnitude (to values in the tens or hundreds of nanometers per millisecond). This arrangement further assures that the volume of the tubing and feeding channel is replaced in minutes. The nanochannels have an height of $200 \mathrm{~nm}$ thereby limiting the movement of the fluorescent molecule to the evanescent field of the TIRF microscope and enabling reliable single-particle tracking (Figure 1b). Another source of spreading of the emitted photons over additional pixels is the movement of the fluorescent molecule due to diffusion; the effect can be strongly reduced by applying a stroboscopic illumination scheme in which the molecule is illuminated only for a fraction of the acquisition time of the frame. ${ }^{[7]}$ Moreover, the illumination of two neighboring frames can be arranged in a back-to-back configuration (Figure 1c) to further minimize the displacements between those frames thus allowing for higher concentrations of molecule in the channels before successful tracking is compromised by overlapping pathways.

\subsection{The Interaction between ARF and Labeled DNA can be Visualized Using smFRET in Nanochannels}

We tested the feasibility of visualizing the interaction between $\mathrm{ARF}$ and its DNA response element in nanofluidic devices by flowing a doubly labeled DNA construct (see also Section 2) through the channels in presence or absence of ARF transcription factors. Upon binding to the DNA construct (Figure 2a), ARF is expected to bend the DNA and sterically confine the movement of the dyes (Figure 2b); thus, ARF binding causes a decrease in the FRET efficiency of the DNA construct. ${ }^{[20,33]}$ Measuring the DNA construct (2.5 $\mathrm{nM}$ in the fluidic devices led to a single population centered at $E^{*}=0.39$ (Figure 2c, top); a second sample containing saturating concentration (256 nM) of MpARF2-DBD showed the characteristic shift toward a lower FRET efficiency $\left(E^{*}=0.31\right.$ , Figure 2c, bottom). The fraction of bound DNA in the sample containing $2.5 \mathrm{nM}$ of MpARF2-DBD was $80 \%$, a value close to the ones obtained in experiments with immobilized DNA samples 85\% (Figure S3, Supporting Information)

We asked to which extend the use of parallel nanochannels allows performing titration experiments without the supervision of an operator. To this end, we opted for sequentially loading the samples containing DNA and increasing concentration of ARF inside the syringe or, alternatively, inside a long connecting tubing. To prevent mixing of neighboring samples, we separated them by an air bubble. This arrangement abolishes dispersing effects created by convection (i.e., Taylor dispersion) while the air bubbles provide a visual cue for the position of the sample. We tested the general feasibility by loading two samples separated by an air bubble into a syringe before feeding the nanochannels. The first sample contained the DNA construct in PBS $1 \times$ buffer (2.5 nM; Figure 2 d,e top) while the second contained both DNA and 256 nM of either MpARF2DBD (Figure 2d, bottom) or MpARF1-DBD (Figure 2e, bottom). The ARF containing samples showed the characteristic shift toward lower FRET efficiencies when compared to the one without ARF; however, the noise present on the $E^{*} S^{*}$ histogram makes the evaluation of the DNA bound fraction inaccurate.

The time duration of an entire titration combined with the presence of air bubbles between the samples makes the use of enzymatic oxygen scavenging system (OSS) problematic; given the presence of molecular oxygen in the sample, we explain the lower quality of the data to be caused by a relevant portion of the counts in the $E^{*} S^{*}$ histogram originating from doubly-labeled DNA molecules in which either the donor or the acceptor fluorophore photobleached during the acquisition of a single frame. 
a

b

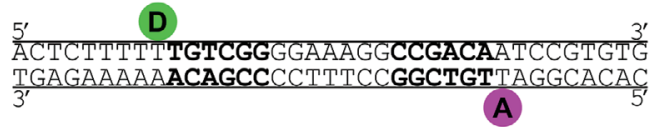

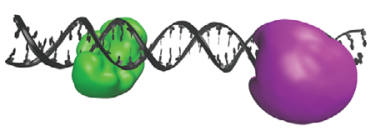

d
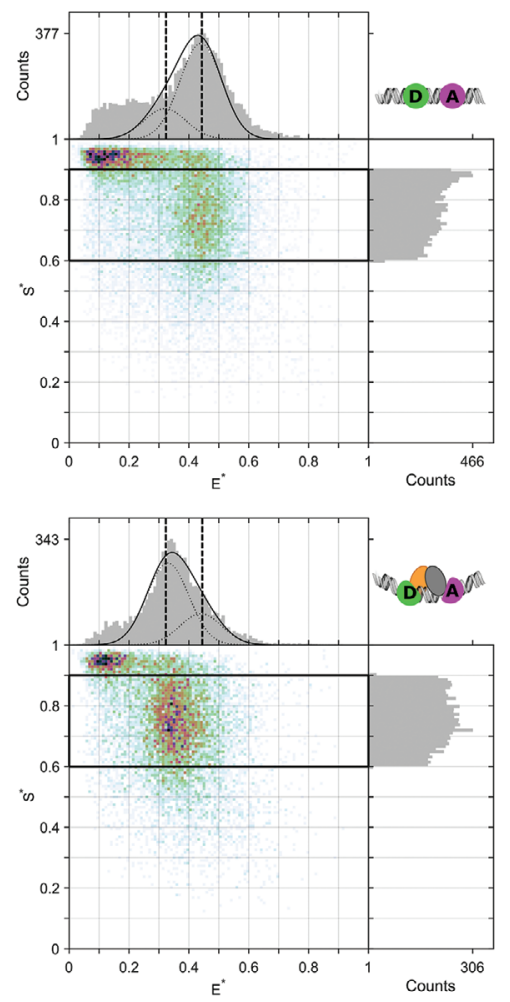

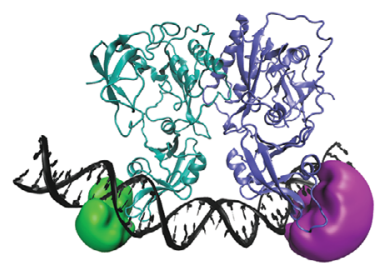

e
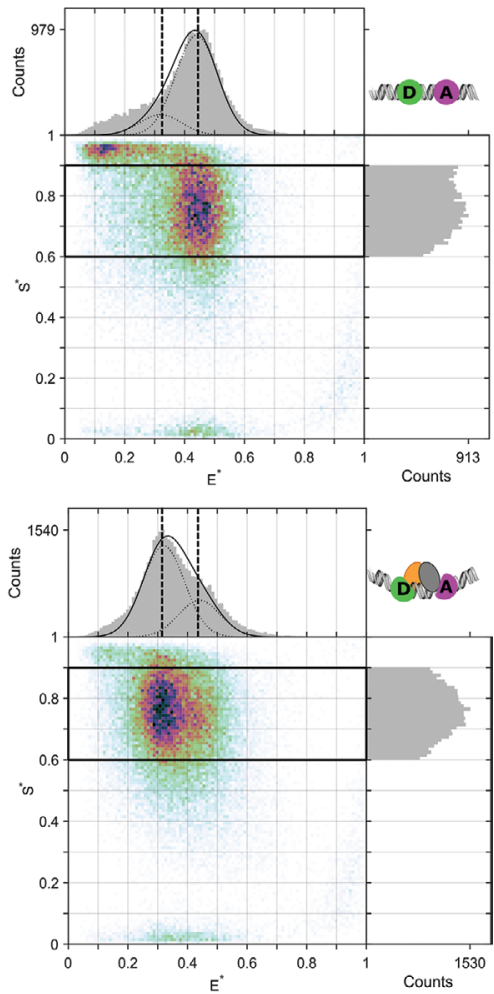

Figure 2. Visualizing ARF-DNA interactions in nanofluidic devices. a) Schematic representation of the doubly-labeled DNA construct with the two binding sites for ARF (bold) and the positions of the donor (D, Cy3B) and the acceptor (A, ATTO647N) fluorophore. b) Accessible volume (AV) simulation for free (left) and ARF-bound (right) DNA construct. The AV represents the volume that can be visited by the dye thanks to its flexible linker. In presence of ARFs, the mean distance between the dyes increases resulting in a decrease of the FRET efficiency $E^{*}$. c) $E^{*} S^{*}$ histograms of a sample containing DNA alone (top) and DNA with a saturating concentration of MpARF2-DBD ( $256 \mathrm{nM}$, bottom). The fraction of bound DNA can be calculated from the relative areas of the two Gaussians centered at the $E^{*}$ of the free and bound DNA. d) The same samples of (c) were loaded consecutively on the syringe while being separated by a air bubble, and without the standard enzymatic oxygen scavenger system being present. The shift in $E^{*}$ upon ARF binding can be seen, but the reduced data quality due to photobleaching does not allow to reliably identify the relative amount of bound and free DNA. e) Same experiment as in (d) but with MpARF1-DBD instead of MpARF2-DBD. Vertical visual guidelines were added in (c-e).

Taken together, these results show that the characteristic shift in FRET efficiency seen in experiments with the surface-immobilized DNA construct can be also seen using freely diffusing DNA. This finding further confirms that the shift in $E^{*}$ is caused by specific ARF to DNA binding rather than surface-induced artifacts (e.g., protein absorption on the surface).

\subsection{Changes in the Diffusion Coefficient of Labeled DNA can be Used to Monitor ARF-DNA Interaction}

In the previous section we described experiments in which doubly-labeled single DNA constructs were imaged and tracked to determine their FRET efficiency $\left(E^{*}\right)$ in absence or in presence of ARF; the resulting change in $E^{*}$ was then used as readout for ARF binding. In this section, we will analyze the change in diffusion coefficient $(D)$ of the DNA constructs to obtain an independent second readout for binding.

During two back-to-back illuminations, the DNA construct in the nanochannels will move due to flow and diffusion. In the direction perpendicular to the flow, the movement is due exclusively to Brownian motion and the distribution of the displacements can be used to calculate the diffusion coefficient ( $D=\sigma^{2} / 2 t$; see also Section 2). The diffusion coefficient of the DNA construct in $1 \times$ PBS buffer (without OSS) was found to be 62(1) and 67(1) $\mu \mathrm{m}^{2} \mathrm{~s}^{-1}$ in two independent experiments 
a
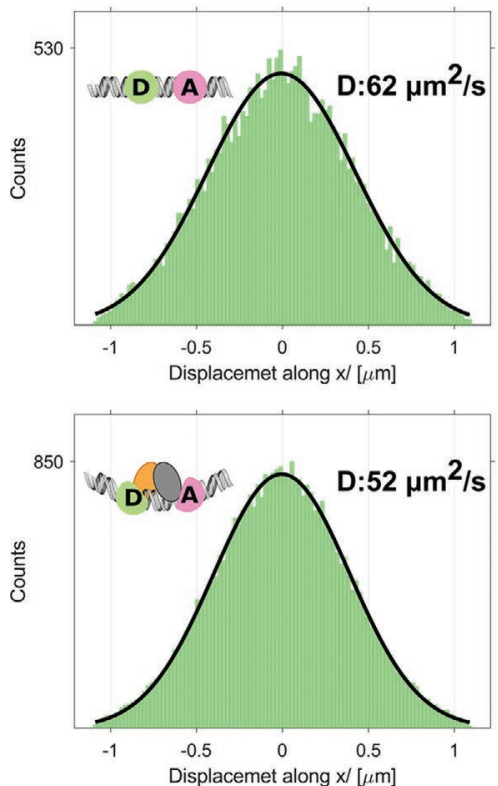

b
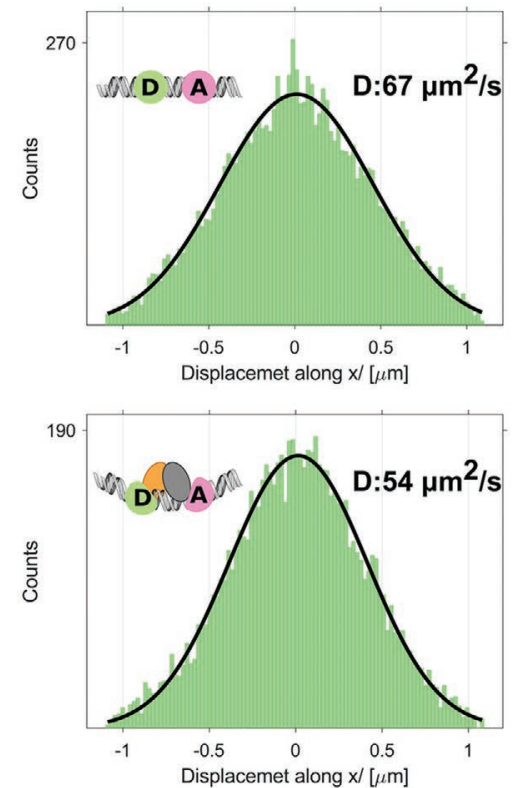

C
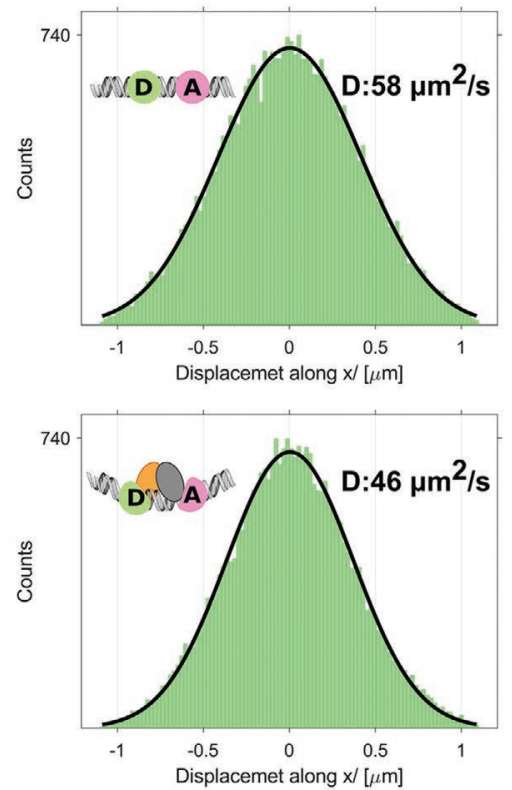

Figure 3. Displacement analysis of the labeled DNA construct in absence or presence of MpARF1-DBD and MpARF2-DBD. a) The diffusion coefficient of the DNA construct decreases by $10 \mu \mathrm{m}^{2} \mathrm{~s}^{-1}$ when $256 \mathrm{nM}$ of MpARF1-DBD is added to the solution, consistent with the formation of a complex of the DNA construct with a protein dimer. b) Same experiment as (a) but using MpARF2-DBD instead of MpARF1-DBD; the starting and final diffusion coefficients are in good agreement with the results in (a). c) Same experiment as in (b) but with the addition of an enzymatic oxygen scavenger system (glucose oxidase/catalase, glucose and Trolox); the decrease in diffusion coefficient upon MpARF2-DBD addition is consistent with (a) and (b) but the absolute values are lower due to the decrease in localization error and an increase of the viscosity of the solution.

(Figure 3a,b, top) and thereby close to the theoretical one of $69.8 \mu \mathrm{m}^{2} \mathrm{~s}^{-1}$ (see Section 2). Upon addition of either MpARF1DBD or MpARF2-DBD, the diffusion coefficient of the DNA construct decreases to 52.3(4) and 54(1) $\mu \mathrm{m}^{2} \mathrm{~s}^{-1}$, respectively (Figure $3 \mathrm{a}, \mathrm{b}$, bottom); these values are again consistent with the theoretical value for the diffusion coefficient of the complex formed by a dimer of MpARF2-DBD bound to the DNA construct which is $51.0 \mu \mathrm{m}^{2} \mathrm{~s}^{-1}$. The analysis of the diffusion coefficients in presence of an OSS showed decreased diffusion coefficients for both free DNA (57.8(9) $\mu^{2} \mathrm{~s}^{-1}$ ) and bound DNA (45.5(6) $\left.\mu \mathrm{m}^{2} \mathrm{~s}^{-1}\right)$. This effect is likely to be caused by the increased viscosity of the sample when the OSS is present in solution. The presence of $1 \%$ glucose alone increases the viscosity by around $2 \% .{ }^{[34]}$ Similar to the experiments without the OSS, addition of ARF decreased the diffusion coefficient of the DNA by around $12 \mu \mathrm{m}^{2} \mathrm{~s}^{-1}$.

\subsection{ARF-DBDs at Nanomolar Concentration are Monomeric}

A common feature of ARF-DBDs as portrayed in crystal structures is that they form dimers caused by interactions between their dimerization domains (DD). Unfortunately, the structures do not provide information on the dimerization equilibrium in solution. To the best of our knowledge, the only data available (acquired using small angle X-ray scattering) reported $\approx 60 \%$ dimer when $78 \mu \mathrm{M}$ Arabidopsis thaliana ARF1-DBD was added in solution. ${ }^{[18]}$ This places a dissociation constant of the dimer to be $\approx 10 \mu \mathrm{M}$. Given the scarcity of information about ARF dimerization in solution we decided to perform experiments in parallel nanochannels. We imaged stochastically labeled AtARF1-DBD and AtARF5DBD $(\approx 1 \mathrm{nM})$ whilst applying the back-to-back stroboscopic illumination scheme ${ }^{[15]}$; the proteins were tracked and the distributions of displacements were determined (Figure 4).

Despite the passivation of the glass surfaces with PEG, a small but noticeable fraction $(<20 \%)$ of single-molecule localization came from ARFs that were adsorbed on the surface; for this reason a two Gaussian fit was employed, in which one Gaussian fits the displacements of the free flowing fraction and the second one the apparent displacements of the adsorbed population. Fitting the distribution of displacements perpendicularly to the direction of flow (along $x$, Figure 4, top) resulted in two Gaussian distributions centered at zero. The wider Gaussian belongs to the free-flowing molecules and its width is proportional to the diffusion coefficient of the freeflowing species while the narrow one belongs to the adsorbed population and its width is equal to double the localization error for an immobile particle (see Section 2). An apparent diffusion coefficient for the adsorbed population was calculated and subtracted from one of the free-moving particles to account for the localization error; we note that although moving particles are expected to have a higher localization error due to motion blur, we used stroboscopic illumination which minimizes this contribution. We obtained diffusion coefficients of 78(2) $\mu^{2} \mathrm{~s}^{-1}$ for AtARF1-DBD labeled with Cy5, 65(2) $\mu^{2} \mathrm{~s}^{-1}$ for AtARF5DBD labeled with ATTO647N and 69.2(7) $\mu \mathrm{m}^{2} \mathrm{~s}^{-1}$ for AtARF5DBD labeled with Cy3B. These values can be compared with the theoretical ones for monomeric and dimeric AtARF5-DBD 
ARF1-DBD Cy5
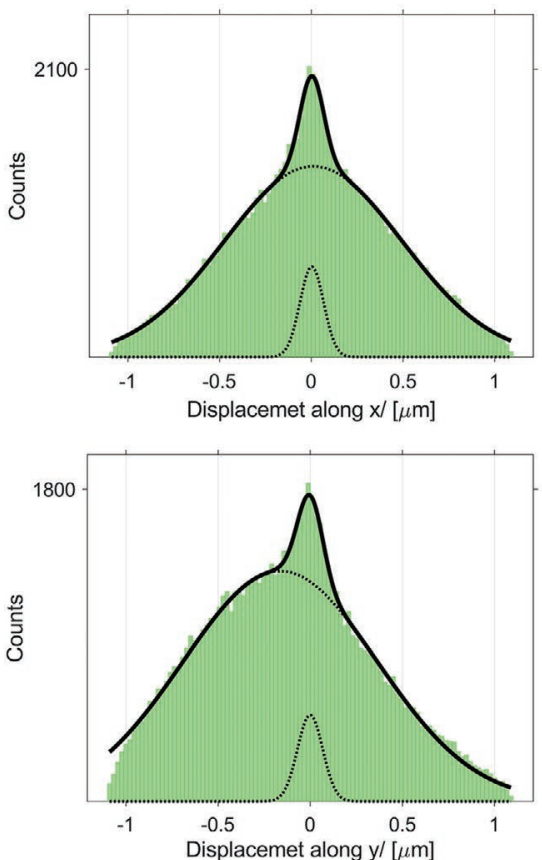

ARF5-DBD ATT0647N
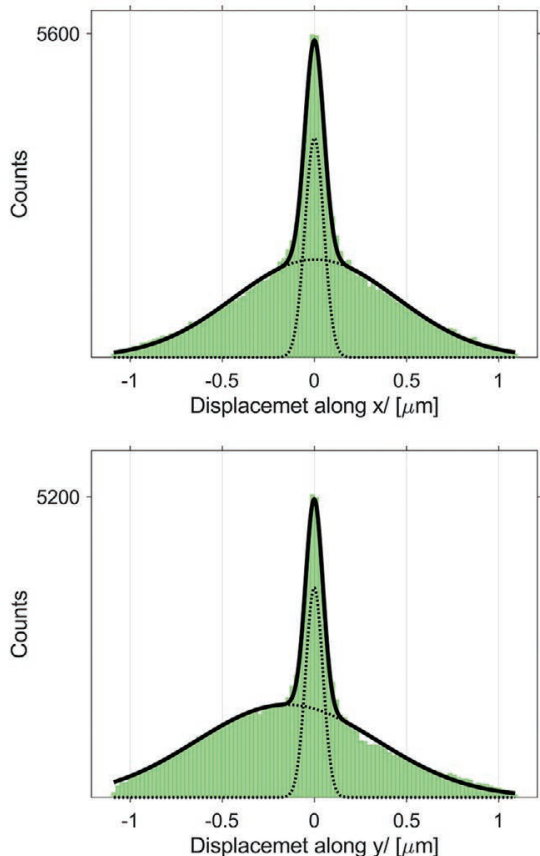

ARF5-DBD Сузв
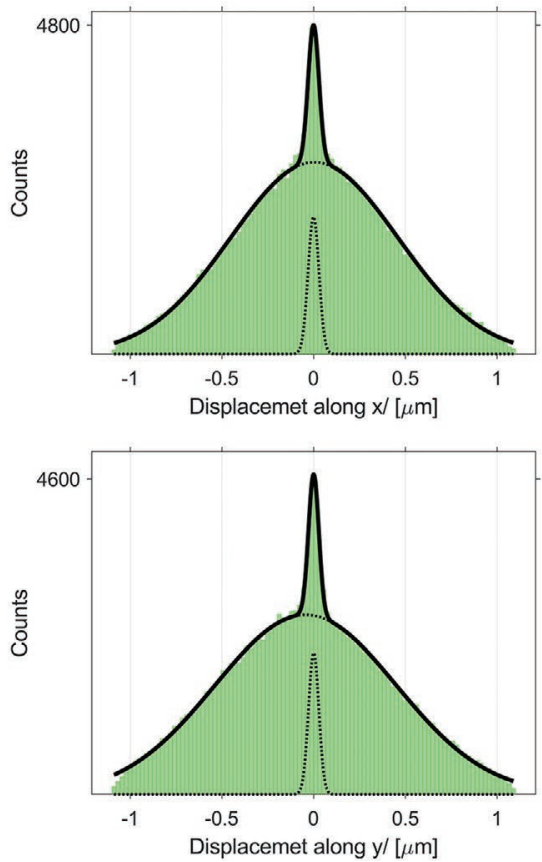

Figure 4. Displacements analysis for AtARF1-DBD (Cy5), AtARF5-DBD (ATTO647N), and AtARF5-DBD (Cy3B) imaged using the single-color excitation scheme. The displacements perpendicular to the flow (along $x$, top) and parallel to the flow (along $y$, bottom) are best fitted with two Gaussians. The slow population (narrow Gaussian) belongs to the proteins absorbed to the surface of the channel as it appears centered around zero even along the direction of flow. The width of the Gaussian fit for this population equals double the localization error and was used to correct the diffusion coefficient $D$ of the freely moving population (wide Gaussian). The resulting diffusion coefficient along $x$ was $78(2) \mu \mathrm{m}^{2} \mathrm{~s}^{-1}$ for AtARF1-DBD labeled with Cy5, 65(2) $\mu \mathrm{m}^{2} \mathrm{~s}^{-1}$ for AtARF5-DBD labeled with ATTO647N and 69.2(7) $\mu \mathrm{m}^{2} \mathrm{~s}^{-1}$ for AtARF5-DBD labeled with Cy3B.

(extracted from the crystal structures PDBID:4LDU, see Section 2) of 73 and $55 \mu \mathrm{m}^{2} \mathrm{~s}^{-1}$, respectively (given the high similarity in structure and size, the values obtained for AtARF1-DBD using the crystal structure PDBID:4LDV showed no difference when rounded to two significant digits). The comparison of the simulated and experimental values for the diffusion coefficient suggests that, at the concentration tested $(\approx 1 \mathrm{nM})$, both AtARF1DBD and AtARF5-DBD are present as monomers in solutions.

Fitting the distribution of displacements along the direction of the flow (along $y$, Figure 4, bottom) shows that the narrow distribution is still centred around zero while the wide population of the freely-moving particles is centered around a value which is proportional to the mean flow speed in the field of view $(\langle v\rangle$ , see Section 2); this proves that the narrow distribution arises from absorbed molecules and not diffusing proteins aggregates. In general, the lack of a narrower population centered around the mean flow speed proves that protein aggregation, if present, is negligible. We note that the diffusion coefficient of the moving particle along $y$ is affected by additional broadening due to the inhomogeneous velocity field inside the channels and cannot be used to determine the diffusion coefficient directly.

\section{Discussion}

In this work, we used single-molecule fluorescence detection performed in fluidic devices to study the DNA binding domain of several members of the family of auxin response factor (ARF) transcription factors and the interaction between them and their DNA response element.

We found that the interaction between doubly-labeled dsDNA bearing the response element and unlabeled ARF led to changes in both the FRET efficiency and the diffusion coefficient. Notably, the readout via a change in diffusion can be compared to the theoretical values for the DNA in the bound and unbound states. Furthermore, monitoring the diffusivity serves as an internal quality control check as potential surfaceinduced artifacts can easily be identified. We further investigated the possibility of performing titrations without the intervention of an operator. The samples were loaded sequentially inside the syringe and were separated using a bubble of air. This approach impeded the use of standard enzymatic oxygen scavenging systems which in turn reduced the overall data quality; nevertheless, the characteristic reduction in FRET efficiency and diffusion coefficient in the samples containing ARF-DBDs was seen. In future experiments, the samples could be loaded in an inert environment (e.g., inert gas atmosphere), to suppress the presence of oxygen between the samples and enable the usage of effective oxygen scavenger systems.

The diffusion coefficients $D$ of stochastically labeled AtARF5DBD and AtARF1-DBD were then used to determine their state of oligomerization in solution. The values found were compatible with a situation in which the DBDs are present mainly as monomers whereas in crystal structures ARF-DBDs are 
always present as dimers. This apparent discrepancy serves as a stark reminder that static images of crystal structures, although extremely useful, fail to capture the dynamic nature of biological processes. We further note that the analysis of the displacements along the flow inside the nanochannels allows detecting protein aggregation, if present, which would lead to a slowly diffusing population characterized by advection. As for the individuation of possible surface-induced artifacts, this is a quality control of the sample that is embedded into the methodology.

The main technical limitation of smFRET measurements in nanofluidic devices come from the limited photon budget and photostability of individual fluorophores. Whereas the influence of motion blur on the localization precision can be accounted for, ${ }^{[35,36]}$ the calculation of FRET values depends on collecting a sufficient number of photons per frame. We note, that using cropped field of views or sCMOS cameras approaching framerates of $1000 \mathrm{~Hz}$, would allow skipping the stroboscopic excitation. Further, the use of single-photon avalanche diode arrays (recently reviewed in ref. [37]) is an interesting concept combining superior time resolution and sensitivity for counting individual photons with high-throughput detection.

\section{Conclusion}

In this work we used SMFD inside parallel nanochannels to study the interaction between ARF-DBDs and between ARFDBDs and their genomic response element. We showed that our approach is able to report on ARF binding by detecting changes in the FRET efficiency similar to experiments with immobilized DNA samples, while obtaining additional information in the form of the displacement distributions. The analysis of this distribution is able to identify the population of absorbed molecules and to separate it from the population of potential aggregates. Moreover, the obtained diffusion coefficient can be used to infer the nature of the tracked molecule/ complex. Taken together, our approach allowed us: i) to check for surface induced artifacts and sample quality during the experiment; ii) to confirm the results coming from changes in $E^{*}$ with a second readout (D); and iii) to infer the oligomerization state of ARF-DBDs in solution. In general, the combination of information from smFRET measurements together with displacement analysis makes SMFD inside nanochannels a powerful method to study protein-protein and protein-DNA association and dissociation.

\section{Supporting Information}

Supporting Information is available from the Wiley Online Library or from the author.

\section{Acknowledgements}

The authors would like to thank all their colleagues at the Laboratory of Biophysics and the Laboratory of Biochemistry for helpful discussions. This work was supported by an Erasmus+ fellowship to S.I., a Ph.D. fellowship (M.F.) from the Graduate School Experimental Plant Sciences to J.H. and D.W. and a VICl grant (No. 865.14.001) from the Netherlands Organization for Scientific Research (NWO) to D.W.

\section{Conflict of Interest}

The authors declare no conflict of interest.

\section{Author Contributions}

The general research questions were proposed by D.W. and J.H. J.H., K.M., and M.F., proposed the methodology and defined the experimental design. S.L. and W.v.d.B. purified and labeled the proteins. S.I., E.v.d.W., and M.F. performed the experiments. M.F. wrote and adapted the software for data analysis and analyzed the data. M.F., J.H., and D.W. discussed the content of the manuscript. M.F. wrote the draft manuscript and produced the figures. M.F., J.H., and D.W. edited and finalized the manuscript.

\section{Data Availability Statement}

The data that support the findings of this study are available from the corresponding author upon reasonable request.

\section{Keywords}

auxin response factor, lab-on-a-chip, microfluidics, single-molecule biophysics, single-molecule fluorescence detection, single-molecule Förster resonance energy transfer, total-internal reflection fluorescence microscopy

Received: May 14, 2021

Revised: July 16, 2021

Published online:

[1] J. Hohlbein, K. Gryte, M. Heilemann, A. N. Kapanidis, Phys. Biol. 2010, 7, 031001.

[2] C. Joo, H. Balci, Y. Ishitsuka, C. Buranachai, T. Ha, Annu. Rev. Biochem. 2008, 77, 51.

[3] E. Lerner, T. Cordes, A. Ingargiola, Y. Alhadid, S. Chung, X. Michalet, S. Weiss, Science 2018, 359, eaan1133.

[4] B. Hellenkamp, S. Schmid, O. Doroshenko, O. Opanasyuk, R. Kühnemuth, S. R. Adariani, B. Ambrose, M. Aznauryan, A. Barth, V. Birkedal, M. E. Bowen, H. Chen, T. Cordes, T. Eilert, C. Fijen, C. Gebhardt, M. Götz, G. Gouridis, E. Gratton, T. Ha, P. Hao, C. A. Hanke, A. Hartmann, J. Hendrix, L. L. Hildebrandt, V. Hirschfeld, J. Hohlbein, B. Hua, C. G. Hübner, E. Kallis, et al., Nat. Methods 2018, 15, 669.

[5] E. Lerner, A. Barth, J. Hendrix, B. Ambrose, V. Birkedal, S. C. Blanchard, R. Börner, H. S. Jung, T. Cordes, T. D. Craggs, A. A. Deniz, J. Diao, J. Fei, R. L. Gonzalez, I. V. Gopich, T. Ha, C. A. Hanke, G. Haran, N. S. Hatzakis, S. Hohng, S.-C. Hong, T. Hugel, A. Ingargiola, C. Joo, A. N. Kapanidis, H. D. Kim, T. Laurence, N. K. Lee, T.-H. Lee, E. A. Lemke, et al., eLife 2021, 10, e60416.

[6] S. Farooq, C. Fijen, J. Hohlbein, Protoplasma 2013, 251, 317.

[7] S. Farooq, J. Hohlbein, Phys. Chem. Chem. Phys. 2015, 17, 27862.

[8] S. Kim, A. M. Streets, R. R. Lin, S. R. Quake, S. Weiss, D. S. Majumdar, Nat. Methods 2011, 8, 242. 
[9] B. Wunderlich, D. Nettels, S. Benke, J. Clark, S. Weidner, H. Hofmann, S. H. Pfeil, B. Schuler, Nat. Protoc. 2013, 8, 1459.

[10] S. Tyagi, V. VanDelinder, N. Banterle, G. Fuertes, S. Milles, M. Agez, E. A. Lemke, Nat. Methods 2014, 11, 297.

[11] J.-Y. Kim, C. Kim, N. K. Lee, Nat. Commun. 2015, 6, 6992.

[12] E. Boukobza, A. Sonnenfeld, G. Haran, J. Phys. Chem. B 2001, 105, 12165.

[13] Q. Wang, W. E. Moerner, J. Phys. Chem. B 2012, 117, 4641.

[14] S. R. Leslie, A. P. Fields, A. E. Cohen, Anal. Chem. 2010, 82, 6224.

[15] M. Fontana, C. Fijen, S. G. Lemay, K. Mathwig, J. Hohlbein, Lab Chip 2019, 19, 79.

[16] B. Gilboa, B. Jing, T. J. Cui, M. Sow, A. Plochowietz, A. Mazumder, A. N. Kapanidis, Biophys. J. 2019, 117, 2141.

[17] T. Ulmasov, G. Hagen, T. J. Guilfoyle, Science 1997, 276, 1865.

[18] D. R. Boer, A. Freire-Rios, W. M. van den Berg, T. Saaki, I. Manfield, S. Kepinski, I. López-Vidrieo, J. Franco-Zorrilla, S. de Vries, R. Solano, D. Weijers, M. Coll, Cell 2016, 156, 577.

[19] D. Weijers, D. Wagner, Ann. Rev. Plant Biol. 2016, 67, 539.

[20] H. Kato, S. K. Mutte, H. Suzuki, I. Crespo, S. Das, T. Radoeva, M. Fontana, Y. Yoshitake, E. Hainiwa, W. van den Berg, S. Lindhoud, K. Ishizaki, J. Hohlbein, J. W. Borst, D. R. Boer, R. Nishihama, T. Kohchi, D. Weijers, Nat. Plants 2020, 6, 473.

[21] I. Rasnik, S. A. McKinney, T. Ha, Nat. Methods 2006, 3, 891.

[22] T. Cordes, J. Vogelsang, P. Tinnefeld, J. Am. Chem. Soc. 2009, 131, 5018.

[23] M. van Dijk, A. M. J. J. Bonvin, Nucleic Acids Res. 2009, 37, W235.

[24] S. Kalinin, T. Peulen, S. Sindbert, P. J. Rothwell, S. Berger, T. Restle, R. S. Goody, H. Gohlke, C. A. M. Seidel, Nat. Methods 2012, 9, 1218.
[25] T. D. Craggs, M. Sustarsic, A. Plochowietz, M. Mosayebi, H. Kaju, A. Cuthbert, J. Hohlbein, L. Domicevica, P. C. Biggin, J. P. K. Doye, A. N. Kapanidis, Nucleic Acids Res. 2019, 47, 10788.

[26] S. J. Holden, S. Uphoff, J. Hohlbein, D. Yadin, L. Le Reste, O. J. Britton, A. N. Kapanidis, Biophys. J. 2010, 99, 3102.

[27] J. C. Crocker, D. G. Grier, J. Colloid Interf. Sci. 1996, 179, 298.

[28] A. N. Kapanidis, N. K. Lee, T. A. Laurence, S. Doose, E. Margeat, S. Weiss, Proc. Natl. Acad. Sci. U. S. A. 2004, 101, 8936

[29] J. Hohlbein, T. D. Craggs, T. Cordes, Chem. Soc. Rev. 2014, 43, 1156.

[30] A. Ortega, D. Amorós, J. García de la Torre, Biophys. J. 2011, 101, 892.

[31] H. Liang, W. J. Nam, S. J. Fonash, NSTI Nanotech 2008, Nanotechnol. Conf. Trade Show, Tech. Proc. 2008, 3, 281283.

[32] K. Mathwig, D. Mampallil, S. Kang, S. G. Lemay, Phys. Rev. Lett. 2012, 109, 118302.

[33] A. Freire-Rios, K. Tanaka, I. Crespo, E. van der Wijk, Y. Sizentsova, V. Levitsky, S. Lindhoud, M. Fontana, J. Hohlbein, D. R. Boer, V. Mironova, D. Weijers, Proc. Natl. Acad. Sci. U. S. A. 2020, 117, 24557.

[34] CRC Handbook of Chemistry and Physics (Ed: W. M. Haynes), 97th ed., CRC Press, Boca Raton, FL 2016.

[35] C. L. Vestergaard, P. C. Blainey, H. Flyvbjerg, Phys. Rev. E 2014, 89, 022726 .

[36] C. L. Vestergaard, J. N. Pedersen, K. I. Mortensen, H. Flyvbjerg, Eur. Phys. J. Spec. Top. 2015, 224, 1151.

[37] C. Bruschini, H. Homulle, I. M. Antolovic, S. Burri, E. Charbon, Light: Sci. Appl. 2019, 8, 87. 